\title{
Coarticulation of Jaw Movements in Speech Production: Is Context Sensitivity in Speech Kinematics Centrally Planned?
}

\author{
David J. Ostry, ${ }^{1}$ Paul L. Gribble, ${ }^{1}$ and Vincent L. Gracco ${ }^{2}$ \\ 1McGill University, Montréal, Québec, Canada H3A 1B1, and 2Haskins Laboratories, New Haven, Connecticut 06511-6695
}

Coarticulation in speech production is a phenomenon in which the articulator movements for a given speech sound vary systematically with the surrounding sounds and their associated movements. Although these variations may seem to be planned centrally, without explicit models of the speech articulators, the kinematic patterns that are attributable to central control cannot be distinguished from those that arise because of dynamics and are not represented in the underlying control signals. We address the origins of coarticulation by comparing the results of empirical and modeling studies of jaw motion in speech. The simulated kinematics of sagittal-plane jaw rotation and horizontal jaw translation are compared with the results of empirical studies in which subjects produce speech-like sequences at a normal rate and volume. The simulations examine both "anticipatory" and "carryover" coarticulatory effects. In both cases, the results show that even when no account is taken of context at the level of central control, kinematic patterns vary in amplitude and duration as a function of the magnitude of the preceding or following movement, in the same manner as that observed empirically in coarticulation. Because at least some coarticulatory effects may arise from muscle mechanics and jaw dynamics and not from central control, these factors must be considered before drawing inferences about control in coarticulation.

Key words: articulator movements; jaw movements; speech kinematics; sensorimotor process; mandible; context sensitivity
Speech production is a sensorimotor process in which neural representations of language are transformed into vocal tract motion. The sounds of speech may be combined in various ways, and the associated articulator movements may vary as the kinematic context changes. This kinematic variation, known as coarticulation, is one of the most pervasive characteristics of speech production. Some aspects of coarticulation may be centrally planned, whereas others may not be planned but may arise from factors such as muscle mechanics, musculoskeletal geometry, and jaw dynamics. Without explicit models of the speech articulators, however, kinematic patterns that are attributable to central control cannot be distinguished from those that arise because of muscle properties and jaw dynamics and are not represented in the central control signals.

In the present paper, we explore the possible origins of kinematic patterns of context sensitivity by comparing the results of empirical and modeling studies of human jaw motion. The sinulations show that even when no account of context is taken at the level of central control, the kinematics may vary as a function of the preceding or following movement. The main point we wish to make is that unplanned effects may arise because of articulator mechanical and dynamic factors, and these must be accounted for before drawing conclusions about the role of central control in coarticulation.

Coarticulation at the level of movement kinematics has been reported in a number of speech articulators and in various experimental manipulations (Kent and Moll. 1969, 1972; MacNeilage

Received Aug. 3, 1995; revised Nov. 29, 1995; accepted Dec. 14, 1995.

This research was supported by National Institutes of Health Grant DC-00594 from the National Institute on Deafness and Other Communication Disordcrs, NSERC-Canada, and FCAR-Québec. The authors thank J. S. Perkell for comments.

Correspondence should be addressed to David J. Ostry, Department of Psychology, McGill University, 1205 Dr. Penfield Avenue, Montréal, Québec, Canada H3A 1B1.

Copyright (C) 1996 Society for Neuroscience $0270-6474 / 96 / 161570-10 \$ 05.00 / 0$ and DeClerk, 1969; Carney and Moll, 1971; Sussman et al., 1973; Barry and Kuenzel, 1975; Butcher and Weiher, 1976; Gay, 1977). Our own work in this area has involved a series of studies in which comparable manipulations have been carried out for the lips, tongue dorsum, velum, and lower pharyngeal wall (Parush et al., 1983; Parush and Ostry, 1986, 1993; Gracco, 1994; also see, Kent and Moll, 1972; Sussman et al., 1973). The strategy has been to select speech stimuli that produce variations in movement amplitude before or after a vocal tract closure. This is typically achieved by manipulating the vowels in vowel-consonant-vowcl (VCV) sequences. In each case, the findings have been similar. We have observed that the position of the articulator (typically its elevation) during consonant production is inversely related to the movement amplitude associated with the preceding and following vowels. That is, the articulator position or elevation for consonant production decreases progressively as the movement amplitude increases for the preceding or following vowel. Consider as an example the patterns of tongue dorsum movement when subjects produce aka versus aku. As the amplitude of the tongue-lowering movement for the final vowel increases (as in $a$ versus $u$ ), the amplitude and duration of the initial tongue-raising movement decrease. Large-amplitude tongue-lowering movements for the final vowel are associated with small-amplitude (and duration) raising movements for the initial vowel-consonant (VC) transition. The coarticulatory effect arises because the tongue-lowering movement starts earlier for lower final vowels (Parush et al., 1983; also see Discussion).

Coarticulatory patterns may be characterized as either "anticipatory" or "carryover." Carryover coarticulation may be observed when movement changes occur after different initial conditions. Anticipatory coarticulation is observed as a result of differences in the composition of the upcoming sequence. Historically, different explanations have been offered for the two forms of coarticulation. Anticipatory coarticulation is presumed to involve explicit 
adjustments to account for upcoming context, whereas carryover effects have been attributed to articulator mechanics.

Initial investigations of coarticulation suggested that anticipatory effects may extend over as many as four intervening consonants (Daniloff and Moll, 1968; Amerman et al., 1970; Benguerel and Cowan, 1974). These observations led to a number of models of speech production in which a "look-ahead" or "scanning" mechanism was proposed (Henke, 1966; Fromkin, 1971; Lindblom and Rapp, 1973; Nooteboom and Cohen, 1975; Keating, 1988). As a group, these models presume that coarticulation is planned. An alternate view has been that coarticulatory changes result from the temporal overlap of control signals associated with the production of vowels and consonants (Fowler, 1977; Hardcastle, 1985; Browman and Goldstein, 1986; Marchal, 1988; SaltzInan and Munhall, 1989). This perspective has been advanced on both the basis of methodological considerations (Gelfer et al., 1989) and the empirical observations, which showed that anticipatory effects are restricted to neighboring phonetic segments (Öhman, 1967; Gay, 1977; Bell-Berti and Harris, 1979; Fowler, 1980; Bell-Berti and Harris, 1981; Boyce et al., 1990). These models suggest that coarticulation arises from the superposition of control signals.

Presumably, other factors such as muscle properties, musculoskeletal geometry, and jaw dynamics also influence coarticulation. These factors should help determine the form of coarticulation, but they are not dependent on explicit contextual adjustments nor do they result from overlapping control signals. The model presented in the next section will be used to explore the extent to which this set of factors contributes to coarticulation in speech.

\section{JAW MODEL}

We have recently proposed a model of jaw and hyoid motion based on the equilibrium point (EP) hypothesis of motor control (Laboissière et al., in press). The model includes neural control signals and reflexes, muscle mechanics, realistic musculoskeletal geometry, and jaw and hyoid bone dynamics. The modeled mechanical properties include the dependence of force on muscle length and velocity, reflex damping, and graded force development attributable to muscle kinetics.

The EP hypothesis proposes that movements result from shifts in the equilibrium state of the system, which arise as a result of changes in the value of neurophysiological control variables that act at the level of the motoneurone (MN) pool. The neural control signals are ultimately mapped onto individual muscles and correspond to a central specification of threshold muscle length $(\lambda)$ for $\mathrm{MN}$ activation. According to the model, force develops in proportion to the difference between the actual nuscle length and the centrally specified threshold muscle length $\lambda$ and the rate of muscle length change. Thus by shifting $\lambda$, the system may move to a new equilibrium position. (For a review of behavioral and physiological evidence supporting the $\lambda$ model, see Feldman, 1986; Feldman et al., 1990; Laboissière et al., in press; Perrier et al., in press.)

The jaw and hyoid model includes seven modeled muscle groups and four kinematic degrees of freedom: sagittal plane jaw rotation, horizontal jaw translation, hyoid vertical translation, and hyoid horizontal translation (Fig. 1). Consistent with empirical data, neural control signals to individual muscles $(\lambda s)$ are coordinated to achieve independent changes in the values of the system's four degrees of freedom (Bothorel, 1975; Ostry and Munhall, 1994). Thus, control is organized to produce movements such as jaw rotation or translation, either alone or in combination.

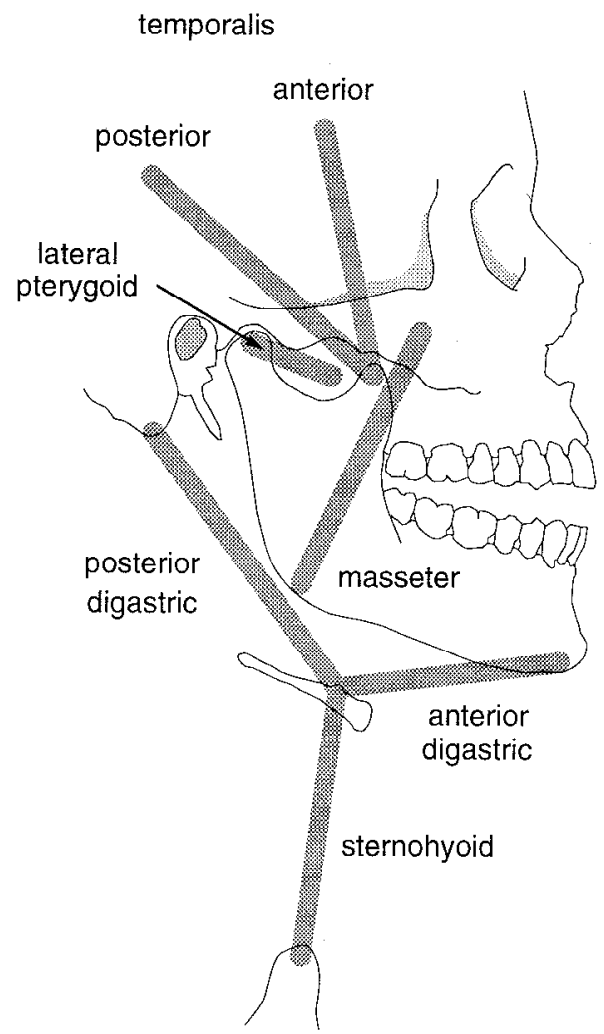

Figure 1. Schematic of the modeled muscle groups and their attachments to the jaw and hyoid bone.

In simulations with the model, we have shown that the smooth empirical patterns of jaw rotation and translation in speech can be achieved using constant rate shifts in the underlying equilibrium orientation and equilibrium position of the jaw. In the tests presented here, the observed kinematic patterns of coarticulation are simulated likewise on the basis of constant-rate equilibrium shifts. The observed kinematic patterns of jaw rotation and translation are compared with simulations of these movements. To assess the extent to which muscle properties and dynamics contribute to the observed coarticulatory patterns, we have held the hypothetical central control signals fixed so that they do not take account of context. Thus at a control level there is no planned coarticulation. We then examine the simulated kinematic patterns to assess whether the empirically observed patterns of coarticulation are nonetheless obtained. This in effect gives us a measure of the contribution of mechanics and dynamics to kinematic coarticulation.

\section{MATERIALS AND METHODS}

Jaw motion kinematics were recorded during the repetitive production of VCV sequences in which the movement amplitudes for the initial and final vowels were varied. The movement amplitudes and durations of the jaw-closing movement associated with the transition between the initial vowel and consonant were assessed as a function of the final vowel (anticipatory coarticulation). Amplitudes and durations of the opening movement from the consonant to the second vowel were assessed with respect to the initial vowel (carryover coarticulation).

The utterances were composed of the vowels $a, o$, and $i$ and the consonants $k$ and $t$. The utterances were embedded between flanking consonants $p$ and $s$ to produce speech-like sequences such as sakas. All combinations of three initial vowels, two consonants, and three final vowels were tested for each of the two flanking consonants. Subjects were instructed to stress both vowels equally and to produce the sequences 


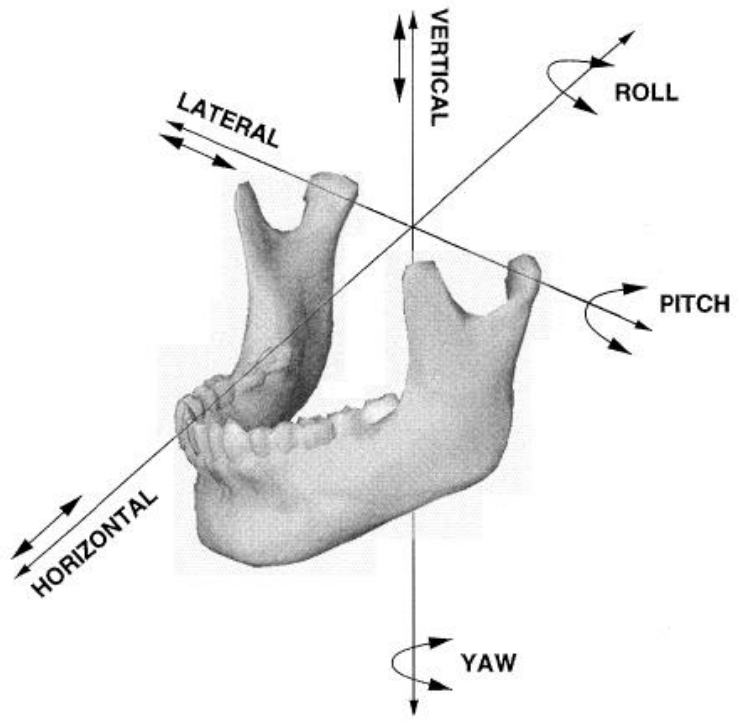

Figure 2. The frame of reference for jaw motion. Note that the HORI$Z O N T A L$ axis is aligned with the occlusal plane.

repetitively at a normal sound level and rate. Approximately 10 samples of each utterance type were recorded. Six speakers were tested.

Jaw motions were recorded at $200 \mathrm{~Hz}$ in three spatial dimensions using Optotrak, an optoelectronic imaging system. The system tracks the motion of infrared emitting diodes (IREDs), which are attached to the jaw and the head. The IREDs on the head (6) were attached to a plastic frame and were used to correct the data to a head-based frame of reference. The jaw IREDs (4) were glued to a light-weight acrylic and metal dental appliance that was attached to the mandibular teeth using a dental adhesive. The appliance was seated bilaterally and custom-molded for each subject to fit the contour of the buccal surface of the teeth.

The three-dimensional raw data for each IRED were low-pass filtered using a second-order zero phase lag Butterworth filter. The cut-off frequency was chosen on the basis of Fourier analysis and through direct comparison of raw and filtered records. A filter frequency of $10 \mathrm{~Hz}$ corresponded to points at which the signal power had dropped $40 \mathrm{~dB}$ from its maximum. Jaw orientation angles and positions were derived using vendor-supplied software. Figure 2 shows the coordinate system in which jaw movements were represented. Jaw positions and orientation angles were characterized with respect to the position of the condyle center at occlusion. The coordinates of this point were obtained by palpation to locate the condyle center and then by measuring the horizontal and vertical distances from that point to a known reference location (tip of the mandibular incisors). The relevant movements were identified by first locating the sound boundaries in the acoustic signal and then locating the corresponding movement start and end on the basis of the tangential velocity of one of the four jaw markers. Because the four markers were rigidly attached to the dental appliance and all were equally close to the mandibular teeth, each of the four markers gave comparable estimates; however, one specified marker was used for all subjects in all cases. Movement start and end were defined as the point closest in value to $0 \mathrm{~cm} / \mathrm{sec}$.

\section{RESULTS}

The basic patterns of jaw motion in speech are shown in Figure 3. It can be seen that the pitch angle, horizontal translation, and vertical translation all contribute significantly to the movement. The roll angle is also seen to vary in a systematic manner; however, its magnitude is small. Our previous work showed that there are essentially two degrees of freedom in jaw movements in speech: the sagittal plane jaw orientation (pitch) and a combination of horizontal and vertical translation (Ostry and Munhall, 1994; Bateson and Ostry, 1995). Jaw motions in these degrees of freedom typically covary. During jaw opening, the jaw rotates downward and translates forward [consonant-vowel (CV) transi-

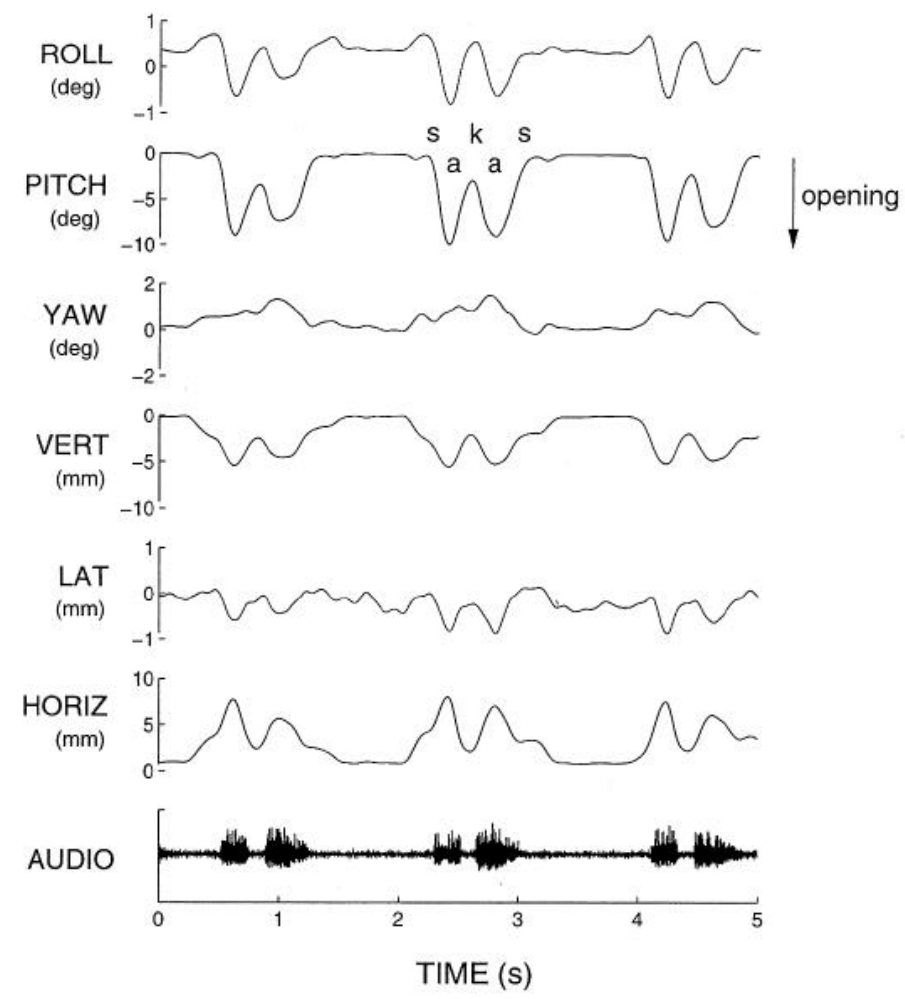

Figure 3. Individual record of repetitions of sakas at normal volume and speech rate.

tion]. During jaw-closing phase, the jaw rotates upward and translates backward (VC transition).

\section{Jaw model predictions}

Anticipatory coarticulation

Figure 4 shows the simulated jaw-motion kinematics (solid lines) and underlying control signals (dashed lines) under three different conditions designed to explore the possible origins of anticipatory coarticulation. In each case, we show a simulated VCV sequence involving jaw closing followed by jaw opening. The simulated kinematics of sagittal plane rotation and horizontal translation are shown.

In the simulations of anticipatory coarticulation (Fig. 4), we have varied the rate and/or duration of the equilibrium shifts associated with the jaw opening movement ( $\mathrm{CV}$ transition) and have held constant the rate and duration of the equilibrium shifts for the initial closing movement. Thus, regardless of the rate or duration of the equilibrium shifts for the final transition, there is a single shift rate and duration for the initial VC closing movement. This is equivalent to varying the identity of the final vowel while holding the initial vowel constant. By examining the simulated kinematics under these conditions, we can assess the extent to which kinematic patterns characteristic of anticipatory coarticulation may arise when at the level of the central control signals no account is taken of upcoming context.

In Figure $4 A$ (left), we see that the rate of equilibrium shift for the final jaw-opening movement (CV transition) is similar for movements of different amplitude, but the shift duration varies. Under these conditions the simulated kinematic patterns of jaw rotation and translation are identical throughout the initial jawclosing phase and diverge only as final position is achieved during jaw opening. 
A
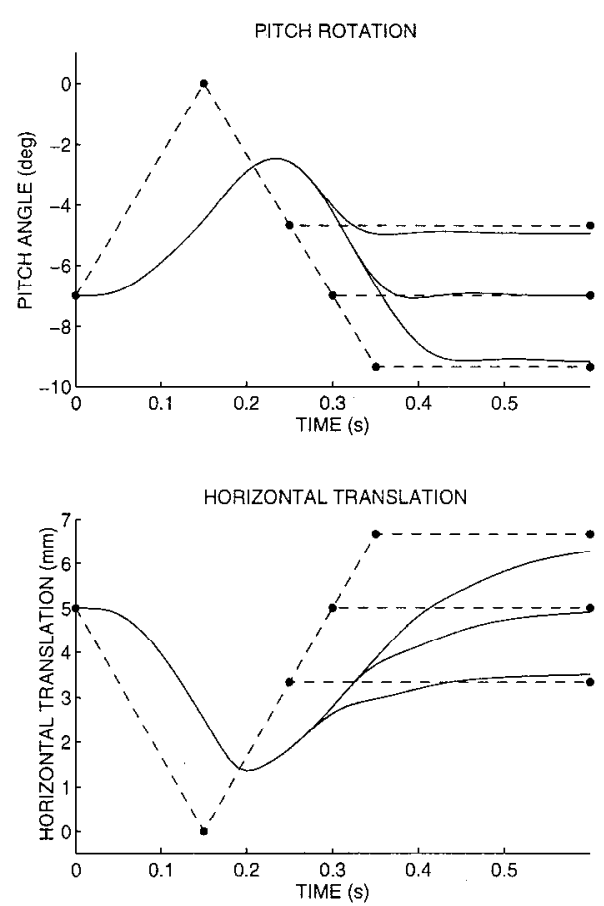

B
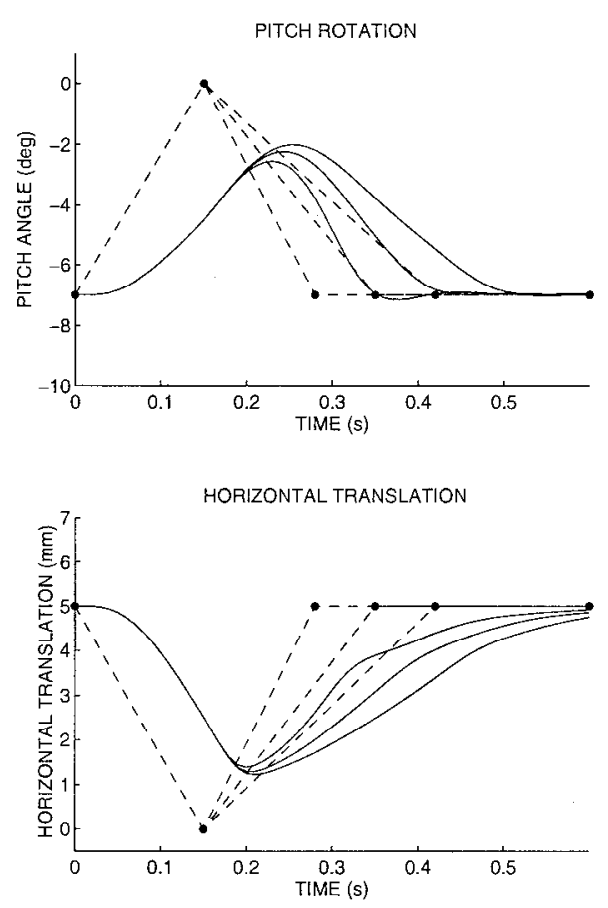

C
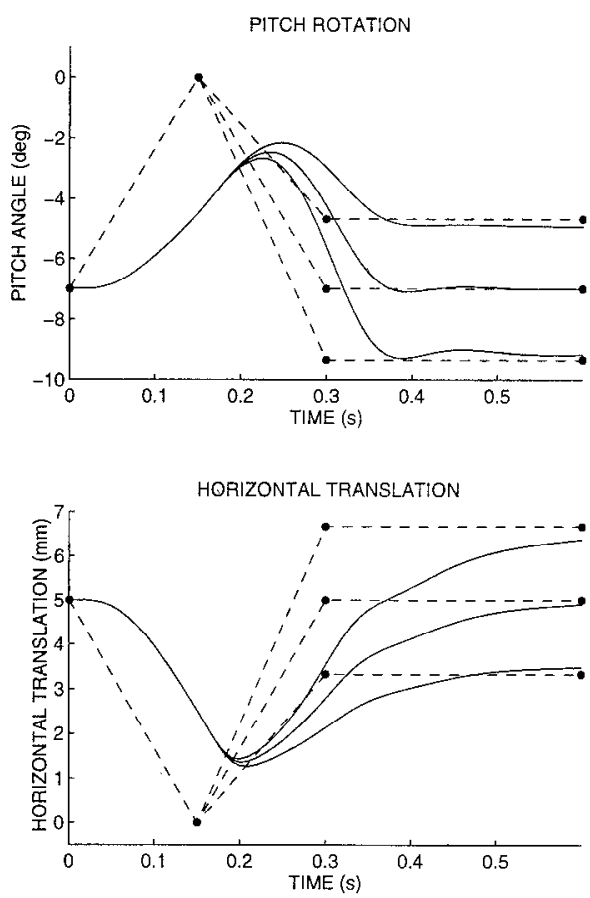

Figure 4. Predicted patterns of jaw rotation and horizontal translation (solid lines) and underlying control signals (dashed lines). The figure shows the possible patterns of anticipatory coarticulation that may arise even when the central control signal for the initial opening movement is the same for all upcoming contexts (see text).

A
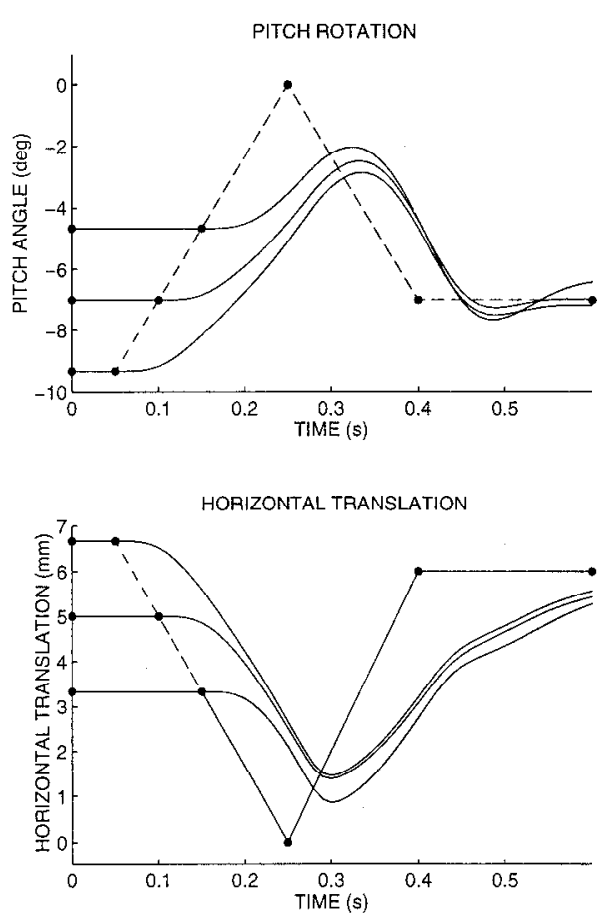

B
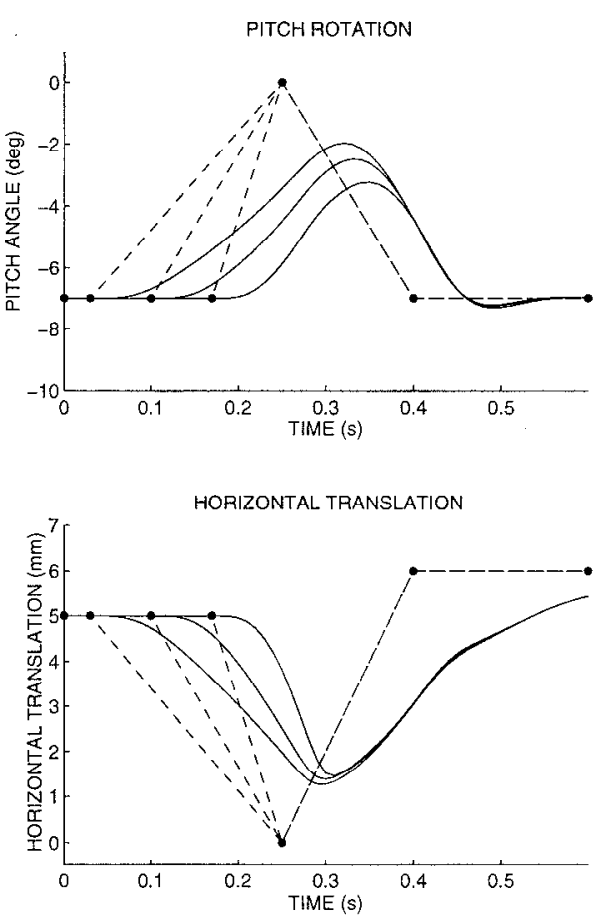

C
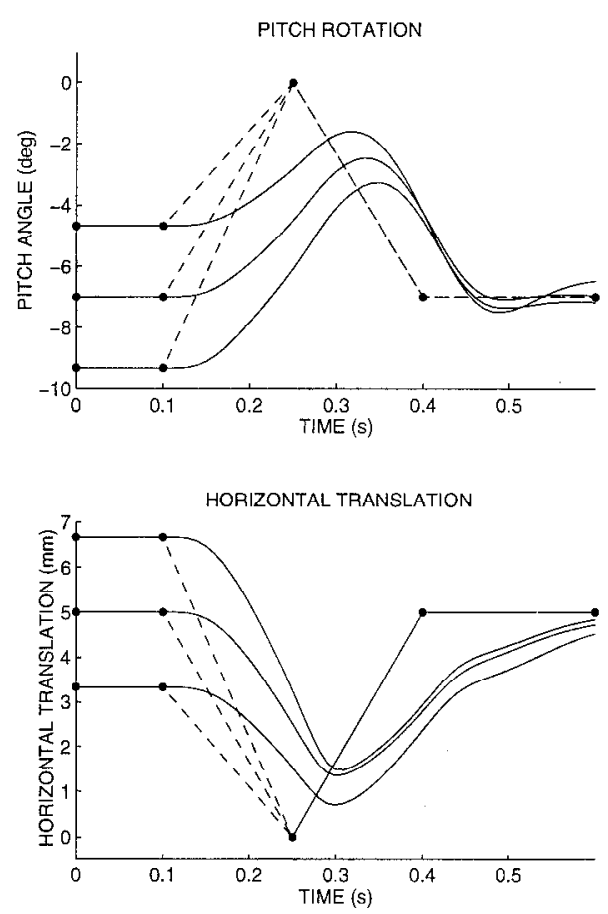

Figure 5. Predicted jaw kinematics (solid lines) and modeled central control signals (dashed lines). The figure shows predicted patterns of carryover coarticulation that may be obtained when the control signal for the final opening movement is fixed (see text for details). 

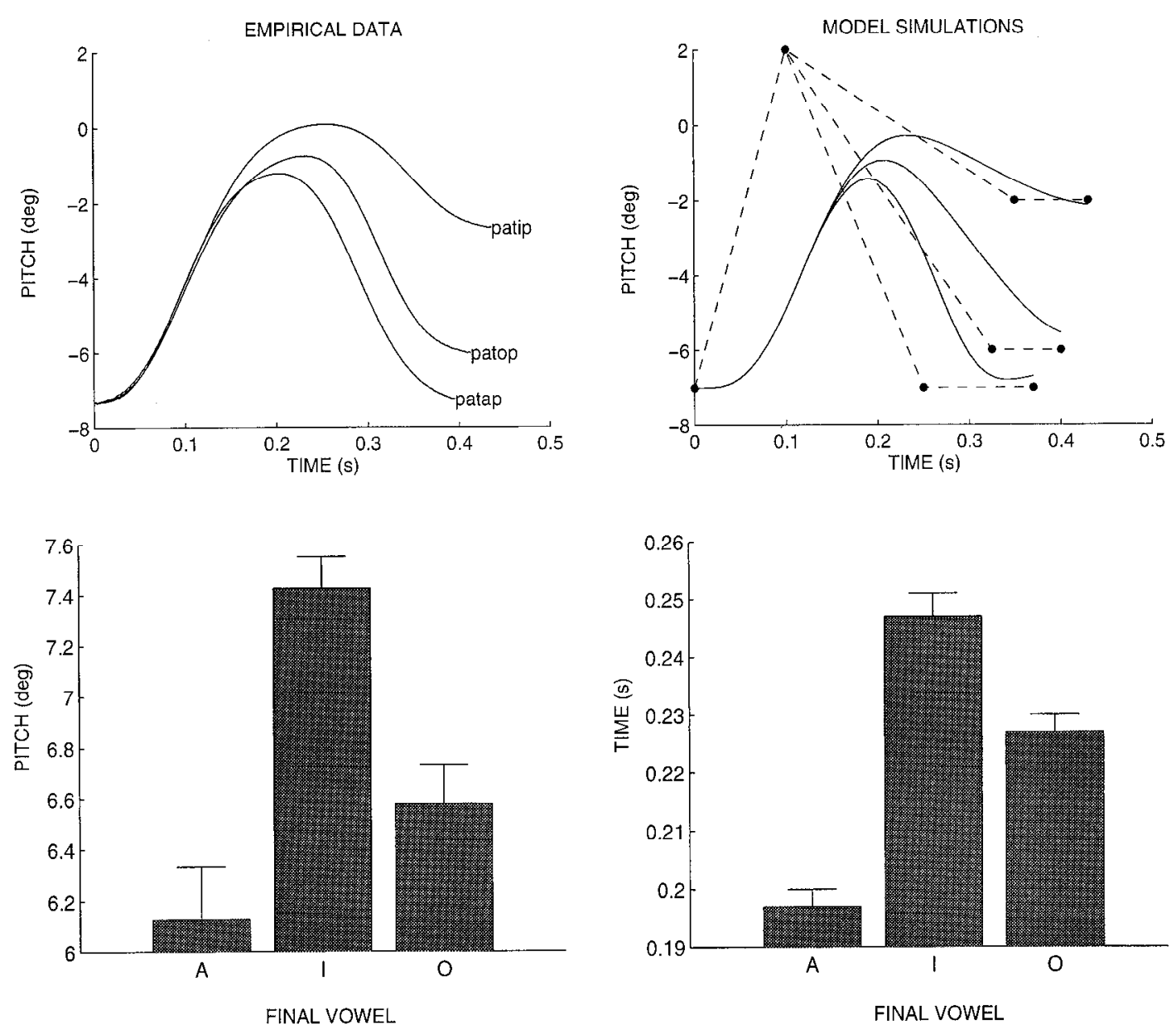

Figure 6. Comparison of empirical data and model predictions for anticipatory coarticulation. Note that kinematic patterns comparable with those observed empirically do not involve any adjustment in central commands with changing phonetic context. The curves shown in the top left are mean data for a single subject in the conditions shown. The curves were time-normalized and aligned for initial position before averaging.

In Figure $4 B$, both the rate and the duration of equilibrium shift associated with jaw opening vary (CV transition) such that movement amplitude is constant. Here, if one were to measure the simulated kinematic amplitude and duration of the initial jawclosing phase (from zero velocity during the initial vowel to zero velocity during consonant closure), it would be seen that the amplitude and duration of the initial movements increase as the speed of the final jaw-opening movement decreases.

In Figure $4 C$, the rate of equilibrium shift associated with jaw opening ( $\mathrm{CV}$ transition) varies, whereas the shift duration is constant. 'I his results in movements of different amplitude but comparable duration. Here again, if one were to measure the kincmatic amplitude and duration of the initial jaw-closing movement, it would be seen that the amplitude and duration of the simulated initial movement increase as the amplitude of the final movement decreases. Here as well, the control underlying the initial movement is not adjusted for changes in upcoming context.

\section{Carryover coarticulation}

Figure 5 shows comparable simulations that examine the simulated jaw motion kinematics of carryover coarticulation. As in the preceding figure, the simulated kinematics (solid lines) and control signals (dashed lines) are shown for three different conditions, which were selected to explore carryover coarticulation. As above, simulated VCV sequences involving jaw closing followed by jaw opening are shown.
For the simulations of carryover coarticulation, the rate and/or duration of the equilibrium shifts for the initial jaw-closing movement are varied, whereas the rate and duration of the shifts underlying the final jaw opening movement are fixed. This is comparable to kinematic studies in which the identity of the initial vowel is varied while the final vowel is held constant. As in our previous example, by examining the simulated kinematics we can assess the extent to which the typical patterns of carryover coarticulation emerge when control signals for the final movement are fixed.

Figure $5 A-C$ ' shows comparable results: the simulated amplitude and duration of the final jaw-opening movement increase as the amplitude of the initial jaw-closing movement decreases. This is the case when the rate of equilibrium shift for the initial movement is fixed $(A)$, when the rate of shift is varied but its amplitude is fixed $(B)$, and when both the rate of shift and the amplitude are varied $(C)$.

\section{Empirical observations}

\section{Anticipatory coarticulation}

The empirical patterns of coarticulation observed here are similar to the patterns predicted in the simulations. Figure 6 provides a summary of the main findings for anticipatory coarticulation using data for pitch as an example. It shows anticipatory coarticulation measured empirically and comparable patterns predicted by the model. Figure 6 (top) shows the average empirical data for a single 

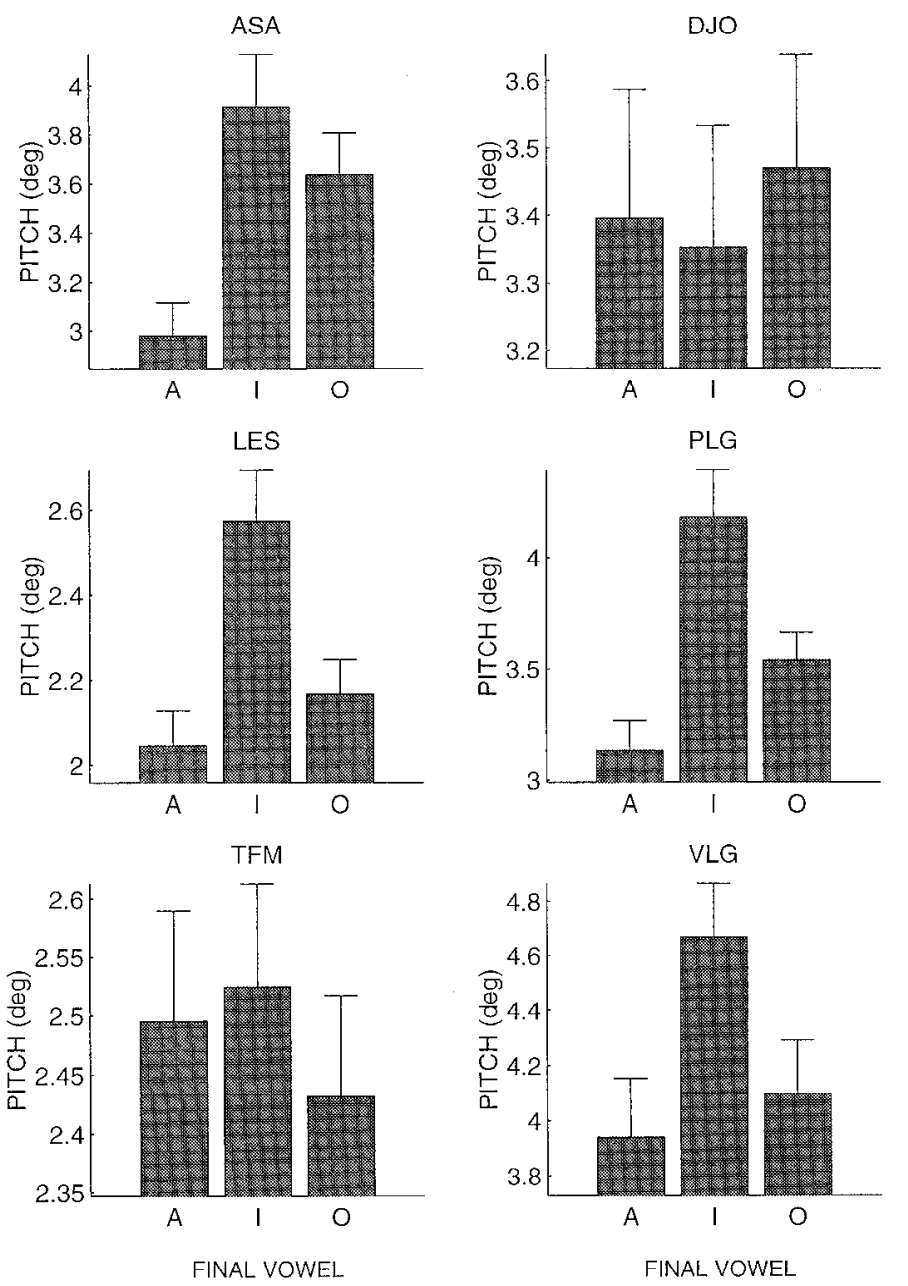

Figure 7. Mean pitch amplitude during jaw-closing movements for different final vowels in VCV utterances (SEs are shown).

subject and comparable simulation results; bottom shows the empirically observed average movement amplitude and duration during the initial jaw-closing phase (VC transition) as a function of the final vowel (for the data at the top left). For visualization purposes, the functions in the top left were time-normalized before averaging and aligned for initial pitch angle. It should be emphasized that the functions shown in the top left are presented as a visual aid. The quantitative results on which our conclusions are based, the mean amplitude and duration of movements for the initial vowel, are shown separately for each subject in the accompanying figures.

Note that as predicted by the simulation (in which the jaw-closing control signal takes no account of upcoming phonetic context), both the average amplitude and the average duration of the initial jaw-closing movement vary inversely with the movement amplitude associated with the final vowel. Initial amplitudes and durations are greatest for the vowel $i$ and least for the vowel $a$.

The jaw-motion patterns were analyzed separately for each subject. In general, subjects followed one of two basic patterns. Either they showed the coarticulatory pattern predicted by the model ( $C$ in Figs. 4 and 5) or their movements showed no effects of context. In a small number of cases, other patterns were observed; however, these were not systematic across subjects.
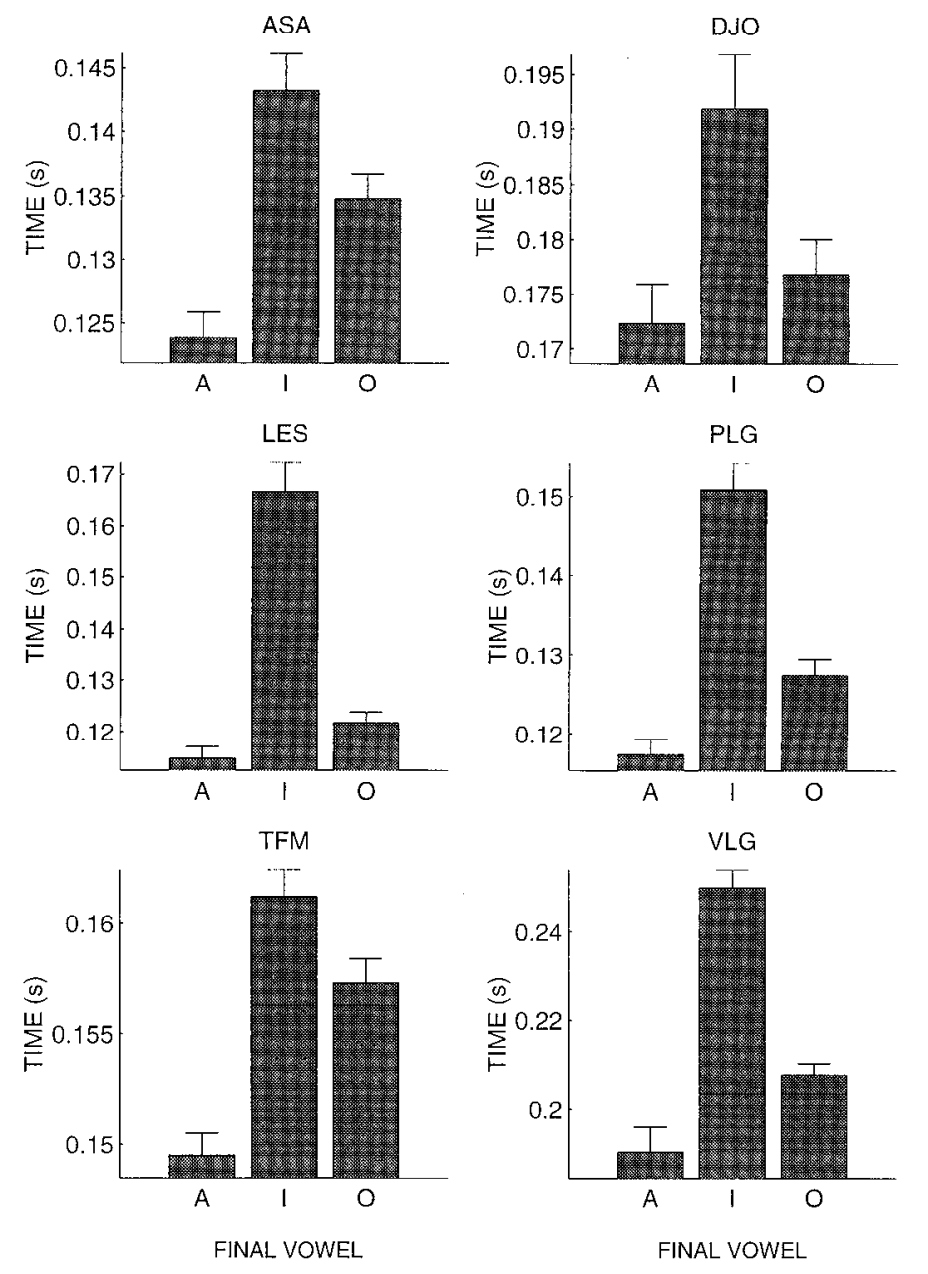

Figure 8. Mean pitch duration during jaw closing for different final vowels (SEs are shown).

We first present a statistical analysis of the anticipatory effects on the sagittal-plane jaw orientation (Figs. 7 and 8). Using ANOVA, the amplitude and duration of the jaw-closing movement for each subject were assessed as a function of the final vowel. Data for different consonants and initial vowels were pooled for these tests. The average pitch amplitude and duration during the initial jaw-closing movement are shown for all subjects as a function of the final vowel. Overall, it can be seen that both the amplitude and the duration of jaw closing are greatest when the final vowel is $i$ and least when the final vowel is $a(p<0.05$ for amplitude; $p<0.01$ for duration); i.e., amplitude and duration vary inversely with movement amplitude for the final vowel. For two subjects, DJO and TFM, the jaw-closing amplitude is similar for all three final vowels $(p>0.05)$.

Anticipatory effects on horizontal jaw translation were obtained for the duration of the translation movement (Fig. 9). For translation durations, the Figure shows two patterns. In one pattern, the duration of translation during jaw closing varies inversely with the movement amplitude for the final vowel $(p<0.05)$. This is consistent with the prediction of the model. Subject PLG shows a different pattern $(p<0.05)$. Systematic patterns were not observed for jaw translation amplitudes (mean differences in translation amplitude in anticipation of different final vowels were $<0.4$ $\mathrm{mm}$ for all subjects). 

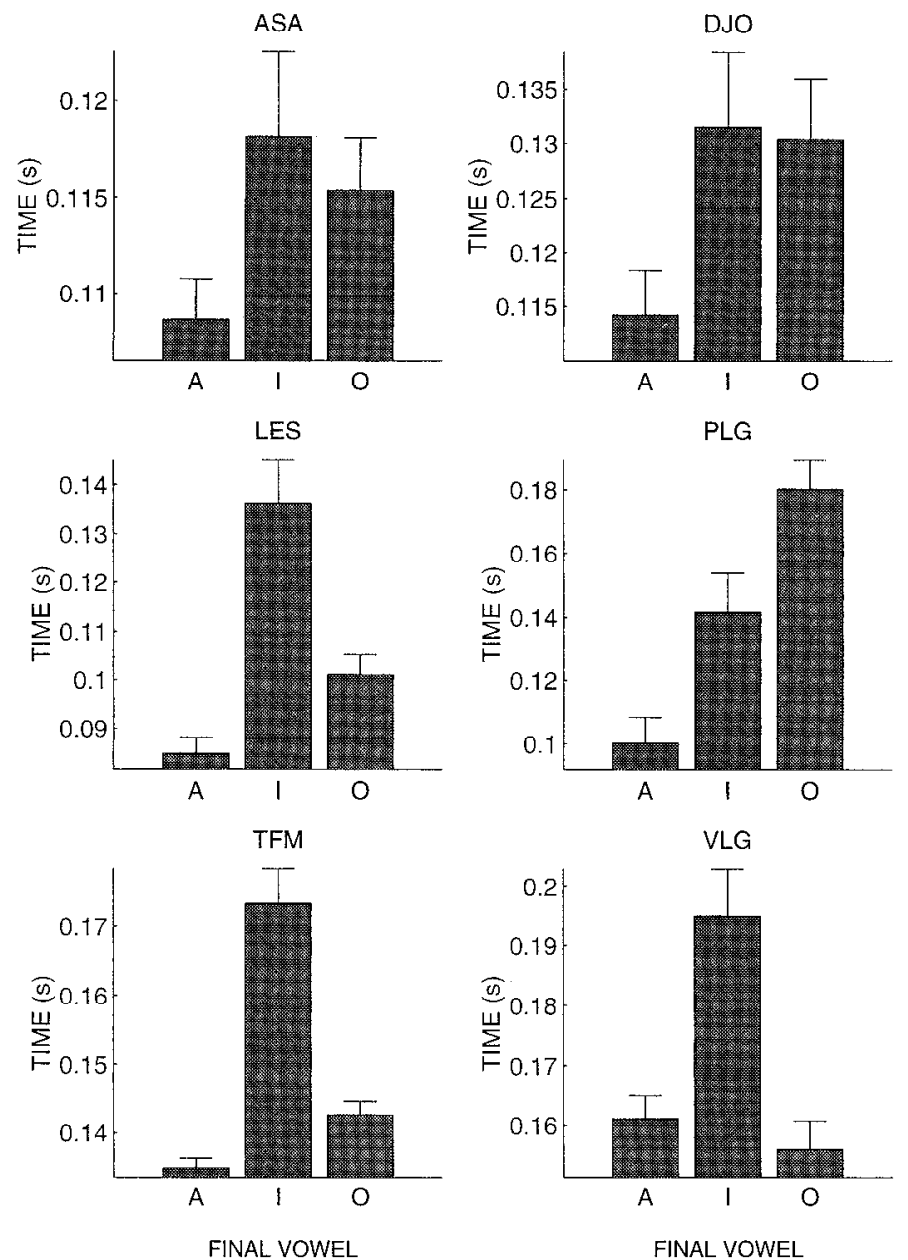

Figure 9. Mean horizontal translation duration during jaw-closing movements as a function of the final vowel (the figure indicates SEs).

\section{Carryover coarticulation}

Figure 10 provides an example of carryover coarticulation. The top shows the average empirical data for a single subject and comparable simulation results, again using pitch as an example. The bottom shows the empirically observed average movement amplitude and duration during the jaw opening as a function of the initial vowel (for the data at the top left). As in Figure 6, for purposes of visualization the functions in the top left were timenormalized before averaging and aligned for final pitch angle. It should be emphasized again that the functions presented in the top left serve primarily as a visual aid. Mean amplitude and duration of carryover movements are shown on a subject-bysubject basis in the accompanying figures.

As in the simulations shown in Figure 5 (in which the jawopening control signal is similar for all initial vowels), the average amplitude and duration of the final jaw-opening movement (from zero velocity during consonant closure to zero velocity during the final vowel) vary inversely with the movement amplitude associated with the initial vowel. Movement amplitudes and durations for the final vowel are greatest when the initial vowel is $i$ and least when it is $a$.

Carryover effects were observed for the duration of jaw rotation and translation (Figs. 11 and 12). In both cases, opening movement durations were greatest when the initial vowel was $i$ and least when the initial vowel was $a(p<0.01$ for both rotation and translation). Thus, as is observed for anticipatory coarticulation, movement duration varies inversely with the movement amplitude for the initial vowel, that is, for the initial jaw closing movement. Note that two subjects, one for rotation (ASA) and one for translation (LES), show other patterns. Systematic carryover effects were not observed for movement amplitudes in either rotation or translation (mean differences in jaw rotation and translation amplitudes after different initial vowels in all cases were $<0.8$ deg and $0.6 \mathrm{~mm}$ ).

\section{DISCUSSION}

We have examined sources of anticipatory and carryover coarticulation in jaw motion by comparing the kinematics of VCV sequences with the results of modeling studies. In the simulations, we have shown that even when no adjustment for changes in context occurs at the level of central control signals, the predicted jaw-motion kinematics differ as a function of context in a manner comparable with that observed in intra-articulator coarticulation. In the modeling studies, these unplanned kinematic effects arise because of muscle properties and jaw dynamics. Accordingly, one should not draw conclusions about the central planning processes underlying coarticulation without explicitly accounting for these factors.

In the simulations, coarticulation arises as a consequence of the forces developed because of equilibrium shifts. As shown in Figure 6 , the centrally specified equilibrium shift that gives rise to the movement occurs well in advance of the accompanying kinematic changes. The initial equilibrium shift toward the consonant position is followed by a subsequent shift back toward the equilibrium configuration for the final vowel. The second shift occurs while the articulator is still in the course of the first movement. The forces and torques that first close the jaw develop in proportion to the difference between the equilibrium and actual jaw positions. When the equilibrium shift begins back toward the final vowel, forces develop in the opposite direction and oppose the initial jaw-closing forces. The magnitude of the opposing forces varies in proportion to the difference between the current equilibrium and actual positions. Thus, as the rate of shift away from the equilibrium configuration for the consonant increases, for example for $a$ versus $i$, the magnitude of the opposing forces also increases. The resulting effect is to reduce the net closing force more for largeamplitude movements than for small movements. This leads to a greater reduction of both the amplitude and the duration of the initial movement toward the consonant for lower final vowels and thus accounts for the variation observed in the simulation results.

The present demonstration that coarticulation in a single articulator may arise from mechanical and dynamical factors rather than from central control cannot be taken as evidence that all instances of coarticulation (even those observed in the jaw) are attributable only to these factors. Although some aspects of coarticulation are attributable to dynamics, other aspects of coarticulation may be attributable to controlled variations (Whalen, 1990). Of course, both factors may influence the same movement. Although it seems that in the present case the system has not adjusted its commands to eliminate differences attributable to dynamics, in other contexts it may do so. The nervous system presumably learns to achieve desired targets by adjusting the control to accommodate dynamics. Although demonstrations of this ability in speech are not known to us, there is ample evidence in other motor systems that sophisticated adjustments for dynamics take place. For example, when an object is grasped and lifted with the fingers, the grip force varies directly in anticipation of the 

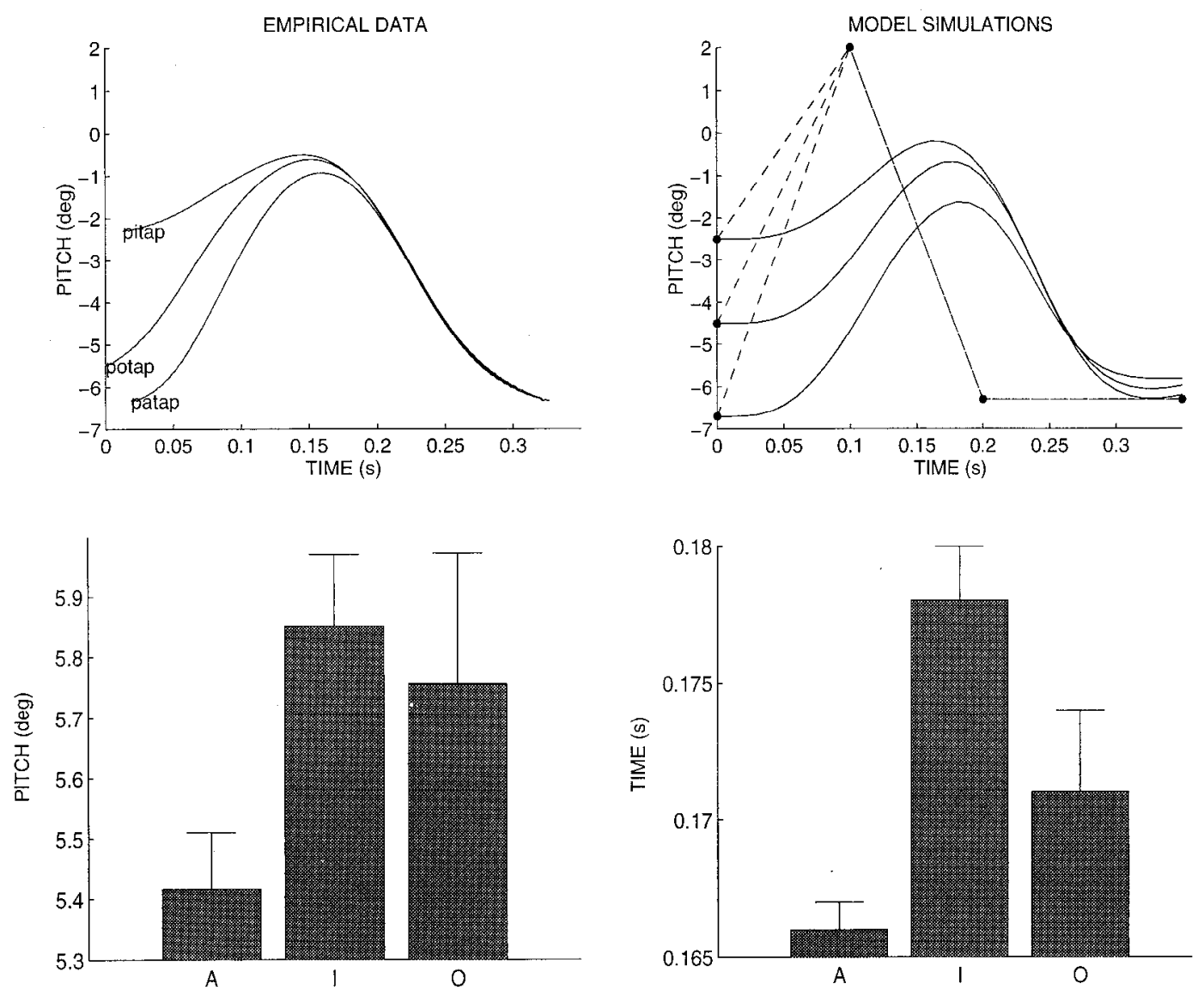

INITIAL VOWEL

INITIAL VOWEL

Figure 10. Comparison of empirical data and model predictions for carryover coarticulation. The top left gives mean data for a single subject. The curves were time-normalized and aligned for initial position before averaging.

load force as determined by the mass and acceleration of the object (Flanagan and Wing, 1993).

The sources of coarticulation revealed in the present study should be distinguished from previous accounts of coarticulation. Because the observed empirical variations may be unplanned, this demonstration should not be equated with the results of so-called "scan ahead" mechanisms (Henke, 1966), nor is the variation observed here equivalent to that in kinematic blending schemes in which it is proposed that coarticulation arises as the result of overlapping control signals for vowel- and consonant-related movements (Fowler, 1977). As the present simulations show, this result is presumably attributable to neither a mixing of commands nor an adjustment for context but rather, as suggested above, to sequential control signals, muscle properties, and jaw dynamics.

A clarification concerning the notion of overlapping of commands may be in order. Note that in the simulations there is no temporal overlap of the control signals themselves. Nevertheless, the control signals do overlap the simulated kinematics. For example, the control signals associated with the jaw-opening movement are initiated before the simulated jaw closure; however, this is not equivalent to overlapping control. Also note that the present demonstration suggests that a single explanation can be offered to account for both anticipatory and carryover coarticulation of the mandible in VCV utterances, as spoken by the subjects in a majority of the cases.

The appropriateness of the $\lambda$ model of jaw movement rests largely on the availability of sources of afferent input to MNs and the effect of that input. (The model suggests that afferent input associated with muscle length and velocity is summed with direct central inputs to $\alpha$ MNs.) Muscle-spindle receptors may provide this information in jaw-closer muscles. In jaw-opener muscles in humans, however, the number of muscle spindles is few. Nevertheless, tonic stretch and unloading reflexes can be recorded in jaw-opener muscles (Lamarre and Lund, 1975; Neilson et al., 1979). We have also recorded in jaw openers both stretch and unloading reflexes, the latter at 10 to $20 \mathrm{msec}$ latencies (our unpublished observations). These demonstrations are consistent with the possibility that afferent input to jaw-opener MNs may arise directly from jaw-opener muscle afferents (including nonspindle afferents). The presencé of a tonic vibration reflex in jaw openers (Hellsing, 1977) indicates that inputs may also arise from mechanoreceptors. Moreover, reflex connections between jawcloser muscles and jaw-opener $\mathrm{MNs}$ in the rat may also provide the necessary afferent input (van Willigen et al., 1986).

A number of features of the model and of the findings should be noted. In Figures 6 and 10, we have shown empirical patterns of coarticulation that correspond to version $C$ of the model in which both the rate and duration of the equilibrium shift were varied (see Figs. 4 and 5). We have used version $C$ for demonstration purposes because it provides the best overall fit to the data. Consistent with empirical observations, it predicts, in addition to the observed coarticulatory patterns, relatively constant 

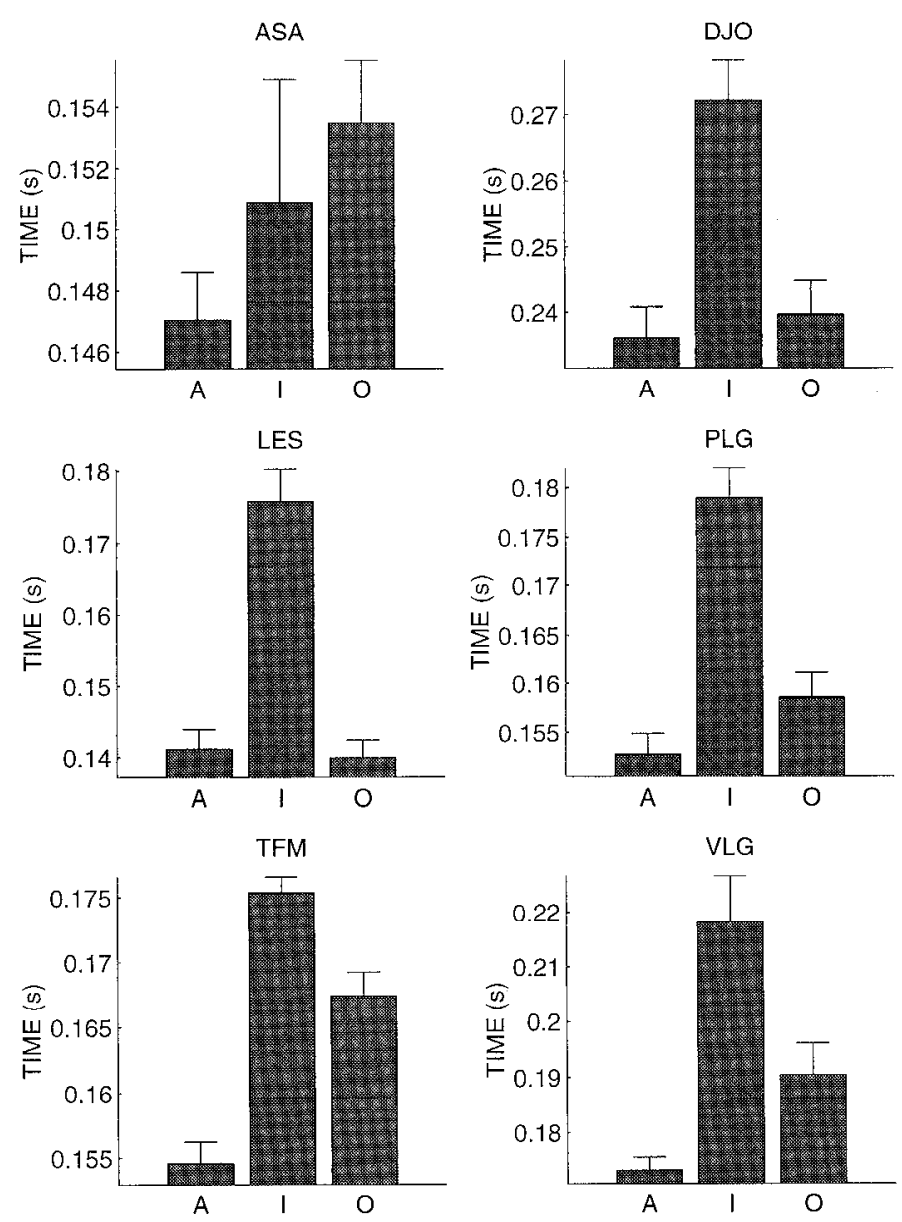

INITIAL VOWEL
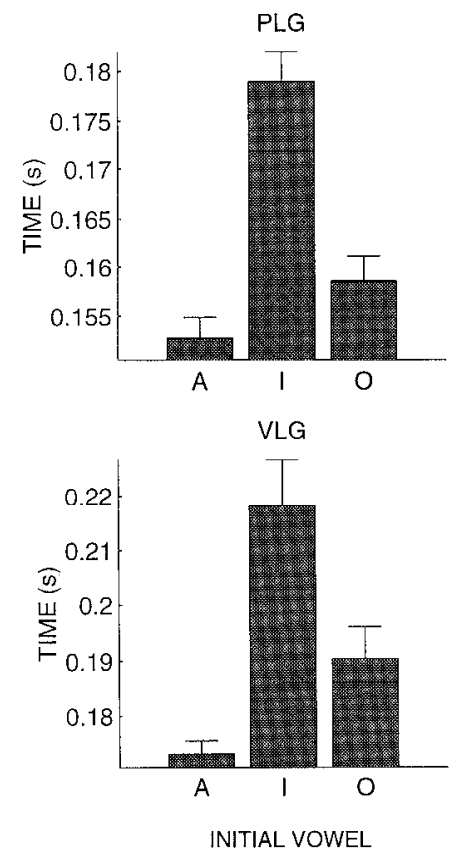
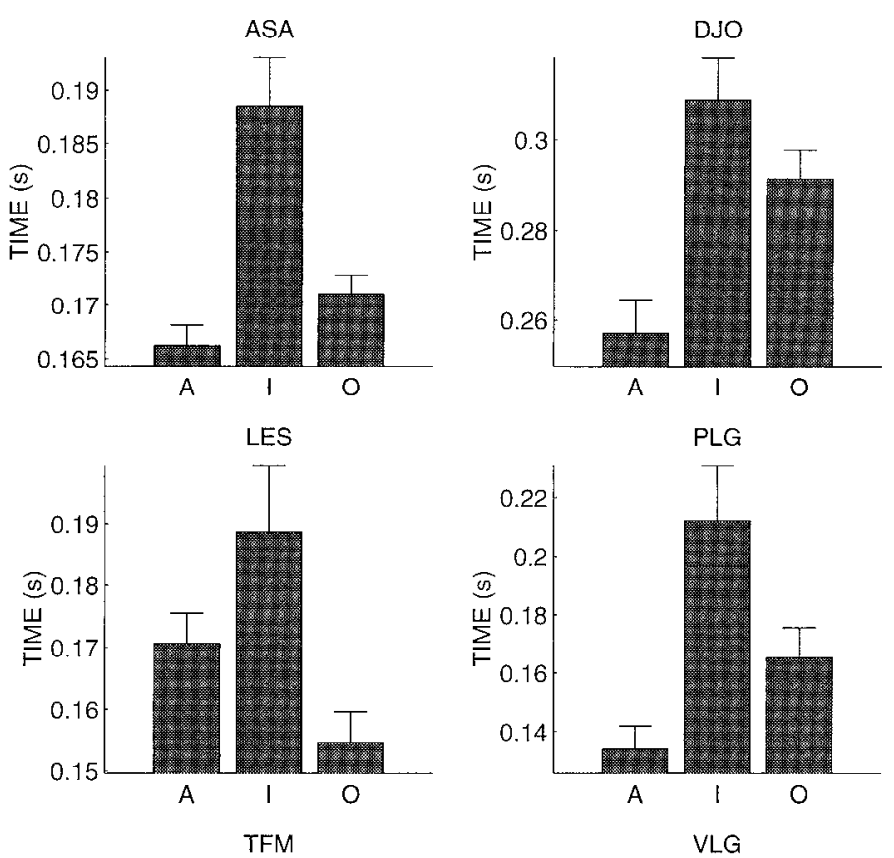

Figure 11. Mean pitch duration during jaw-opening movements for different initial vowels in VCV utterances (SEs are indicated).

movement durations and maximum velocities that increase with movement amplitude.

It should be noted also that coarticulatory effects were absent in some cases. We know of no other empirical studies that have separately examined coarticulatory patterns of jaw rotation and translation and to which the present data might be compared. In the context of the model, however, the absence of coarticulation warrants comment. In order to produce empirical patterns in which no adjustment for context is observed at the level of the simulated kinematics, it is typically necessary to adjust central control signals to eliminate kinematic evidence of coarticulation. Thus, it may be in just those cases in which coarticulation is not observed kinematically that it is in fact centrally planned.

The potential sources of experimental and modeling error require consideration. Subjects have been tested in non-naturalistic speaking conditions using repetitive nonspeech utterances. Although in normal speaking conditions subjects may adjust for changes in context, they may fail to do so herc. Onc should note, however, that the issue is not whether subjects actually adjust for context in a given situation, but rather that unless we are able to separate the effects of dynamics from those of central control, the issue of adjustment for context cannot be resolved. Characteristics of the model itself are potential sources of error. For example, it can be shown that aspects of the presumed control signals, spe-
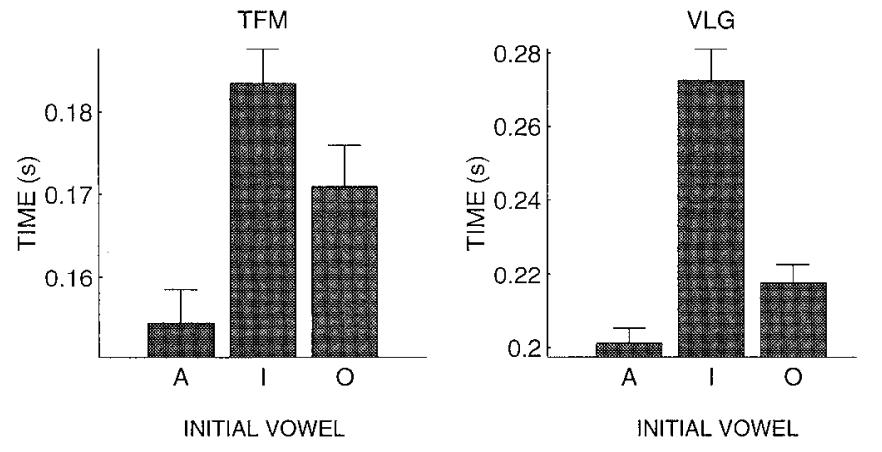

Figure 12. Mean horizontal translation duration during jaw opening for different initial vowels (the figure gives SEs).

cifically the cocontraction level and the form of the equilibrium shifts, affect the simulatcd kincmatics. In control studics carricd out in the preparation of this report, we observed that although changes in these variables do affect individual movements, the overall patterns of coarticulation that we have reported here are not affected. Similarly, geometric and inertial properties of the model, such as the dimensions of the jaw, its mass, and its muscle-attachment points, affect the simulated kinematics. For these variables as well, we have found that the overall patterns of coarticulation are unaffected. It should also be noted that the empirical and modeling data differ in a number of respects. Trial-to-trial variation is observed in individual utterances but is absent in the simulated movements. This is because variability in control signals has not been accounted for in the model, although we have shown elsewhere how variation in jaw kinematics may arise from changes to centrally controlled cocontraction levels (Perrier et al., in press).

By itself, the mandible makes very little direct contribution to determining the characteristics of the sounds that come out of the mouth, with the significant exception of its role as a barrier against which the air-stream is directed to enhance noise generation in certain consonants such as $s$, sh, and possibly the noise burst of $t$. For most sounds, the mandible's main and very important role is to provide a platform for 
movements of the tongue and lower lip. The controlled variables for such movements may include perceptual goals. Thus the commands to the jaw in speech must be coordinated with those to other articulators to achieve desired vocal-tract configurations and the perceptual goals of speech. Coordination may be dependent in part on muscles that the jaw shares with other vocal tract structures and on heteronymous reflex connections (for revicw, sce Dubner ct al., 1978; Kent et al., 1990). In this way, reported phenomena such as perturbations to individual vocal tract structures may lead to adjustments to motions of other structures (Abbs and Gracco, 1983).

Recent experimental studies of human arm movements have raised questions concerning the validity of the EP hypothesis (Lackner and Dizio, 1994). Specifically, it has been suggested that the failure of subjects to achieve correct final limb position in studies of pointing movements in the presence of Coriolis forces violates the notion of equifinality, which is normally associated with the EP model. The errors and the changes to endpoint trajectory during adaptation, however, can be explained by the $\lambda$ model (Feldman et al., 1995). Subjects may decrease the arm deflections attributable to Coriolis forces by curving the equilibrium trajectory of the endpoint in the direction opposite to the curvature of the actual movement. This gives an endpoint error in the direction of the Coriolis force.

\section{REFERENCES}

Abbs JH, Gracco VL (1983) Sensorimotor actions in the control of multimovement speech gestures. Trends Neurosci 6:393-395.

Amerman JD, Daniloff R, Moll KL (1970) Lip and jaw coarticulation for the phoneme /ae/. J Speech Hear Res 13:147-161.

Barry W, Kuenzel H (1975) Co-articulatory airflow characteristics of intervocalic voiceless plosives. J Phonetics 3:263-282.

Bateson EV, Ostry DJ (1995) An analysis of the dimensionality of jaw movement in speech. J Phonetics 23:101-117.

Bell-Berti F, Harris KS (1979) Anticipatory coarticulation: some implications from a study of lip rounding. J Acoust Soc Am 65:1268-1270.

Bell-Berti F, Harris KS (1981) A temporal model of speech production. Phonetica 38:9-20.

Benguerel AP, Cowan HA (1974) Coarticulation of upper lip protrusion in French. Phonetica 30:41-55.

Bothorel A (1975) Positions et mouvements de l'os hyoïde dans la chaine parlée. Trav Inst Phonétique de Strasbourg 7:80-132.

Boyce SF, Krakow RA, Bell-Berti F, Gelfer CE (1990) Converging sources of evidence for dissecting articulatory movements into core gestures. J Phonetics 18:173-188.

Browman CP, Goldstein LM (1986) Towards an articulatory phonology. Phonology Yearbook 3:219-252.

Butcher A, Weiher E (1976) An electropalatographic investigation of coarticulations in VCV sequences. J Phonetics 4:59-74.

Carney PJ, Moll KL (1971) A cineflourographic investigation of fricative consonant-vowel coarticulation. Phonetica 23:193-202.

Daniloff R, Moll KL (1968) Coarticulation of lip rounding. J Speech Hear Res 11:707-721.

Öhman S (1967) Numerical models of coarticulation. J Acoust Soc Am $41: 310-320$.

Dubner R, Sessle BJ, Storey AT (1978) The neural basis of oral and facial function. New York: Plenum.

Feldman AG (1986) Once more on the equilibrium-point hypothesis $(\lambda$ model) for motor control. J Mot Behav 18:17-54.

Feldman AG, Adamovich SV, Ostry DJ, Flanagan JR (1990) The origin of electromyograms: explanations based on the equilibrium point hypothesis. In: Multiple muscle systems: biomechanics and movement organization (Winters J, Woo S, eds.) Berlin: Springer.
Feldman AG, Ostry DJ, Levin MF (1995) Velocity-dependent Coriolis force perturbations: an explanation of positional errors and adaptation. J Neurosci Abstr 21:681.

Flanagan JR, Wing AM (1993) Modulation of grip force will luad force during point-to-point arm movements. Exp Brain Res 95:131-143.

Fowler CA (1977) Timing control in speech production. PhD thesis, Dartmouth College.

Fowler CA (1980) Coarticulation and theories of intrinsic timing. J Phonetics 8:113-133.

Fromkin VA (1971) The non-anomalous nature of anomalous utterances. Language 47:27-52.

Gay T (1977) Articulatory movements in VCV sequences. J Acoust Soc Am 62:183-193.

Gelfer C, Bell-Berti F, Harris K (1989) Determining the extent of coarticulation: effects of experimental design. J Acoust Soc Am 86:2443-2445.

Gracco VL (1994) Some organizational characteristics of speech movement control. J Speech Hear Res 37:4-27.

Hardcastle WJ (1985) Some phonetic and syntactic constraints on lingual coarticulation. Speech Commun 4:247-263.

Hellsing $G$ (1977) A tonic vibration reflex evoked in the jaw opening muscles in man. Arch Oral Biol 22:175-180.

Henke WL (1966) Dynamic articulatory model of speech production using computer simulation. PhD thesis, M.I.T.

Keating PA (1988) Underspecification in phonetics. Phonology 5:3-29.

Kent RD, Martin RE, Sufit RL (1990) Oral sensation: a review and clinical perspective. In: Human communication and its disorders, (Winitz H, ed), pp 135-191. Norwood, NJ: Abeltex.

Kent RD, Moll KL (1969) Vocal tract characteristics of the stop cognate. J Acoust Soc Am 46:1549-1555.

Kent RD, Moll KL (1972) Cinefluorographic analysis of selected lingual consonants. J Speech Hear Res 15:453-473.

Laboissière R, Ostry DJ, Feldman AG Control of multi-muscle systems: human jaw and hyoid movements. Biol Cybern, in press.

Lackner JR, Dizio P (1994) Rapid adaptation to Coriolis force perturbations of arm trajectory. J Neurophysiol 72:299-313.

Lamarre Y, Lund JP (1975) Load compensation in human masseter muscles. J Physiol (Lond) 253:31-35.

Lindblom B, Rapp K (1973) Some temporal regularities of spoken Swedish. PILUS, Stockholm University.

MacNeilage PF, DeClerk JL (1969) On the motor control of coarticulation in CVC monosyllables. J Acoust Soc Am 45:1217-1233.

Marchal A (1988) Coproduction: evidence from epg data. Speech Commun 7:287-295.

Neilson PD, Andrews G, Guitar BE, Quinn PTG (1979) Tonic stretch reflexes in lip, tongue and jaw muscles. Brain Res 178:311-327.

Nooteboom SG, Cohen A (1975) Anticipation in speech and its implication for perception. In: Proceedings of the Symposium on Dynamic Aspects of Speech Perception.

Ostry DJ, Munhall KG (1994) Control of jaw orientation and position in mastication and speech. J Neurophysiol 71:1515-1532.

Parush A, Ostry DJ (1986) Superior lateral pharyngeal wall movements in speech. J Acoust Soc Am 80:749-756.

Parush A, Ostry DJ (1993) Lower pharyngeal wall coarticulation in VCV syllables. J Acoust Soc Am 94:715-722.

Parush A, Ostry DJ, Munhall KG (1983) A kinematic study of lingual coarticulation in VCV sequences. J Acoust Soc Am 74:1115-1125.

Perrier P, Ostry DJ, Laboissière The equilibrium point hypothesis and its application to speech motor control. J Speech Hear Res, in press.

Saltzman E, Munhall KG (1989) A dynamical approach to gesture patterning in speech production. Ecol Psychol 1:1615-1623.

Sussman HM, MacNeilage PF, Hanson RJ (1973) Labial and mandibular dynamics during the production of bilabial consonants: Preliminary observations. J Acoust Soc Am 16:397-420.

van Willigen JD, Juch PJW, Ballintijn CM, Broekhuijsen ML (1986) A hierarchy of neural control of mastication in the rat. Neuroscience 19:447-455.

Whalen DH (1990) Coarticulation is largely planned. J Phonetics 18:3-35. 